\title{
EP-181
}

\section{Curative resection of bladder cancer pancreas head metastasis}

\author{
Sunho OK, Hyungil SEO*, Youngmok PARK, Jaeri KIM, Myunghee YOON \\ Department of Surgery, Pusan National University Hospital, Busan, Korea
}

Introduction: Bladder cancer is the 9th most frequent cancer worldwide and the incidence is increasing. The pancreas is an infrequent site of metastasis in relation to any type of malignancy. In this study, we report our experience with patient who underwent pancreaticoduodenectomy for metastatic bladder cancer.

Methods: In this study, we report our experience with patient who underwent pancreaticoduodenectomy for metastatic bladder cancer.

Results: A 61-year-old man was admitted with jaundice and pancreas head mass. He been received Robot assisted-cystectomy and ileal conduit for bladder cancer 7 months previously. Initial diagnosis under the imaging study was resectable pancreas head cancer but we didn't rule-out the metastatic bladder cancer. He was underwent a classic pancreaticoduodenectomy. Based on the histologic findings and immunohistochemistry result, the pancreas tumor was diagnosed as $4.9 \mathrm{~cm}$ sized metastatic urothelial carcinoma. He experienced no complication and was discharged 11 days after surgery. Four cycles of Gemcitabine and cisplatin were administered and he remained recurrence-free of tumors for 16 months.

Conclusions: The benefit of pancreatectomy in terms of patients of survival has been reported for metastases from renal cell carcinoma, but this is not known in bladder cancer because of no report. We believe that curative resection for metastasis to pancreas of urothelial carcinoma might be helpful to manage. 